A N N A L E S Annales de Bretagne et des Pays de l'Ouest

\title{
La Place des armements mixtes dans la mobilisation de l'arsenal de Brest
}

\section{André Lespagnol}

\section{(2) OpenEdition}

9 Journals

\section{Édition électronique}

URL : http://journals.openedition.org/abpo/2352

DOI : $10.4000 / a b p o .2352$

ISBN : 978-2-7535-1852-0

ISSN : 2108-6443

\section{Éditeur}

Presses universitaires de Rennes

Édition imprimée

Date de publication : 30 mars 2012

Pagination : 207-211

ISBN : 978-2-7535-1850-6

ISSN : 0399-0826

\section{Référence électronique}

André Lespagnol, «La Place des armements mixtes dans la mobilisation de l'arsenal de Brest », Annales de Bretagne et des Pays de l'Ouest [En ligne], 119-1 | 2012, mis en ligne le, consulté le 23 septembre 2020. URL : http://journals.openedition.org/abpo/2352 ; DOI : https://doi.org/10.4000/abpo.2352

Ce document a été généré automatiquement le 23 septembre 2020.

(c) Presses universitaires de Rennes 


\title{
La Place des armements mixtes dans la mobilisation de l'arsenal de Brest
}

\author{
André Lespagnol
}

\section{RÉFÉRENCE}

Jean-Yves Nerzic, La Place des armements mixtes dans la mobilisation de l'arsenal de Brest sous les deux Pontchartrain (1688-1697 \& 1702-1713), Milon-la-Chapelle, Éditions H \& D, 2010, 2 vol., 1288 p. (ISBN 978-2-914266-19-2).

1 Le gros ouvrage dont nous rendons ici compte, constitue la publication intégrale d'une thèse soutenue en 2008 par Jean-Yves Nerzic à l'Université de Bretagne-Sud, avec comme objet l'analyse d'une page originale de l'histoire maritime française sous Louis XIV : les «armements mixtes », c'est-à-dire, selon les termes de l'auteur, «des armements pour lesquels des vaisseaux du roi sont prêtés, pour armer en course, à des particuliers réunis en une société de droit privé qui prennent en charge les frais de fonctionnement ». Il élargit d'ailleurs la notion à l'ensemble des prêts de navires du roi, y compris ceux accordés sans finalité corsaire à des compagnies de commerce, et au roi d'Espagne durant la guerre de Succession pour rapatrier en Europe l'argent américain, ce qui permet de prendre toute la mesure de ce processus de semi-privatisation de la flotte royale durant cette période. Avec une précision cependant quant au périmètre géographique de l'étude, qui est centrée sur la base de Brest, et son annexe de PortLouis - Lorient, en intégrant aussi l'essentiel des armements effectués dans d'autres arsenaux du Ponant, Le Havre et Rochefort, qui fonctionnaient en symbiose avec la grande base bretonne et recevaient son appui technique. Cependant, elle laisse hors champ les armements mixtes effectués dans deux autres arsenaux majeurs, Dunkerque, pionnier en la matière dès le temps de Jean Bart, et Toulon, qui s'y lança intensivement après 1707, avec les armements de Cassard, ce qui limite la vision globale du phénomène. Appuyé sur le dépouillement systématique des correspondances entre le ministre et les responsables des arsenaux du Ponant (intendants, commissaires ordonnateurs), ainsi que des fonds du Conseil des Prises et de la Marine aux Archives 
nationales, ce solide travail nous permet d'éclairer en profondeur les mécanismes complexes de ces armements hybrides.

Son premier apport est de mettre au clair de manière définitive le cadre réglementaire qui a permis l'essor de ce type d'armement, depuis l'ordonnance initiale du 5 octobre 1674 jusqu'au texte décisif du 6 octobre 1694, qui stabilisa le dispositif avec la clause réservant le cinquième du produit des prises au roi, en contrepartie de la mise à disposition de vaisseaux carénés, équipés de leur gréement, artillerie et munitions, à charge pour les partenaires privés de financer les coûts de fonctionnement, c'est-à-dire l'avitaillement et la solde des équipages. L'analyse détaillée des armements montre aussi que ce cadre général a pu être modulé au cas par cas, en fonction de la capacité d'influence des promoteurs privés auprès de la Cour. Le roi élargit encore les avantages accordés aux armateurs privés par le règlement du $1^{\mathrm{er}}$ juillet 1709 , en renonçant à son cinquième sur les prises, en contrepartie d'un financement par ceux-ci de l'équipement matériel des navires prêtés, que l'État, en quasi faillite, n'était plus capable d'assurer. J.Y. Nerzic met aussi en évidence une ouverture supplémentaire vers les investisseurs privés, qui furent autorisés à partir de 1695 à faire construire dans les arsenaux des vaisseaux et frégates pour la course, sous réserve de préfinancer les matériaux nécessaires et les salaires des ouvriers, quitte à s'en rembourser ultérieurement sur le cinquième des prises revenant au roi. Cette possibilité fut utilisée par Nesmond et Saupin dès 1695-97, et par les Trouin après 1704, avec au total une trentaine de navires construits en quinze ans dans les arsenaux royaux. À cet égard, le recours aux armements mixtes a aussi permis de « sauver » les arsenaux et leur potentiel technique et humain menacés par le désengagement croissant d'un État financièrement exsangue.

Dans un second temps, qui occupe les deux tiers de l'ouvrage, J.-Y. Nerzic effectue une analyse "événementielle » détaillée - parfois trop, s'agissant des péripéties des armements ou des litiges lors de la liquidation des prises - de l'ensemble des armements mixtes effectués entre 1688 et 1712 à Brest, et dans les arsenaux « satellites » de Port-Louis-Lorient et Le Havre (et pour partie Rochefort), qui débouche sur un recensement que l'on peut considérer comme exhaustif. Ce travail de fond permet tout d'abord de mesurer l'ampleur, en termes "physiques »- nombre de navires armés, tonnage - de l'effort d'armement ainsi réalisé en partenariat avec des investisseurs privés. Il l'évalue globalement à 254 navires, dont 195 vaisseaux, avec une analyse fine du matériel naval utilisé, révélant un recours privilégié aux vaisseaux de troisième rang, de 48 à 60 canons, qui semblent avoir été les mieux adaptés pour la course. Ces données permettent aussi d'évaluer le tonnage des armements mixtes effectués à Brest : 117675 tonneaux, dont 71650 pour la guerre de la Ligue d'Augsbourg et 46125 pour celle de Succession. Ce sont des chiffres tout à fait considérables, qui permettent de situer la base brestoise au troisième rang des ports d'armement corsaire français entre 1688 et 1713 , après Dunkerque et Saint-Malo, du moins en termes "physiques ", sans poser ici la question de la localisation des centres de décision réels ou de la «domiciliation» du capital. Ces données permettent aussi de retracer l'évolution chronologique de ces armements, en mettant en évidence leur véritable démarrage lors des années 1695-1697 (avec un pic record en 1696: 33400 tonneaux armés!), et un parcours plus heurté durant la guerre de Succession, avec deux pics distincts, en 1705 et 1711 (armement contre Rio). Cette trajectoire des armements pose évidemment la question des facteurs qui peuvent expliquer l'adoption par l'État de cette forme particulière d'utilisation de ses forces navales, et interroge plus 
globalement sur ses choix stratégiques. J.-Y.Nerzic récuse avec vigueur la thèse, dominante depuis Mahan, d'un changement brutal de stratégie navale consécutif à la défaite de La Hougue, conduisant à l'abandon de la guerre d'escadre au profit d'une stratégie de "guerre au commerce ». Il démontre en effet, preuves à l'appui, que Louis XIV a effectivement reconstitué le potentiel de la flotte française dans l'année qui a suivi la défaite, et qu'ont été armées en 1693 (Lagos) et 1694 les plus fortes escadres françaises du temps de la marine à voile. Pour lui, l'élément décisif est la terrible crise économique de 1694, avec ses conséquences sur les finances publiques, qui contraint le roi à abandonner la guerre d'escadre et incitera l'État, financièrement exsangue, à explorer d'autres modes d'utilisation de ses forces navales, parmi lesquelles une grande course cofinancée par les investisseurs privés, scénario qui se reproduit après la crise de 1709. L'on peut cependant penser qu'il y a un lien entre cette " privatisation » de la flotte royale, et la décision stratégique prise par Louis XIV dès l'hiver 1692-93 de renoncer à toute nouvelle tentative de débarquement en Angleterre pour rétablir le roi Stuart : dès lors c'est l'utilité même d'une flotte de premier rang, très coûteuse, qui se trouve mise en cause, comme elle le sera en 1704 après la bataille indécise de VelezMalaga. C'est dans ce contexte d'une véritable impasse stratégique navale, et du repli sur une stratégie de "guerre au commerce ", qu'a pu s'imposer l'idée d'un recours massif aux « armements mixtes » pour la course, en optimisant ainsi le potentiel naval considérable de «l'inutile armada» (pour reprendre l'heureuse formule de Daniel Dessert), avec le renfort bienvenu du capital privé pour financer les coûts de fonctionnement.

4 Troisième apport de ce travail, l'analyse événementielle des armements permet d'éclairer concrètement le rôle des acteurs, quoique de manière inégale entre les deux types de partenaires associés dans ces armements mixtes. Le rôle des acteurs étatiques est celui qui est le mieux mis en évidence, et d'abord celui des acteurs centraux - les ministres et leur entourage, de Seignelay aux Pontchartrain - dont J.-Y. Nerzic souligne le rôle d'impulsion et d'incitation dans le choix des cibles proposées aux armateurs, le rôle décisionnel dans l'attribution des vaisseaux et le choix des capitaines, tout en pointant leurs incohérences, notamment dans les conditions particulières accordées à tel groupe d'investisseurs sous la pression de groupes d'influence. On peut cependant s'interroger sur la capacité réelle du pouvoir central à mettre en œuvre une véritable stratégie globale de "guerre au commerce", et à coordonner l'action des divers armements dont il était partie prenante, au-delà du soutien au coup par coup d'initiatives d'origine externe. Surtout, l'ouvrage souligne le rôle essentiel des représentants du pouvoir sur le terrain, c'est-à-dire les intendants de marine en poste à Brest, Desclouzeaux (de 1683 à 1701) puis Robert (à partir de 1704). Ce sont ces " officiers de plume » de haute volée, agissant à la fois comme chefs de l'administration de la Marine et directeurs des arsenaux, mais aussi comme " contrôleurs opérationnels de zone " - selon l'heureuse expression de l'auteur - dans tout l'espace maritime entre Pas-de-Calais et Gibraltar, qui ont été «du côté de l'État » la cheville ouvrière de ces armements hybrides. Ce sont eux qui, dans un contexte financier de plus en plus tendu, ont vraiment organisé la mobilisation à Brest des moyens matériels et humains (levées de matelots) nécessaires, en les combinant avec ceux apportés par les partenaires privés, non sans tensions quant à l'interprétation des traités signés. J.-Y. Nerzic met ainsi en valeur le rôle et la personnalité de Desclouzeaux, qui a réussi à opérer la reconversion de l'arsenal de Brest après 1694 vers cette nouvelle forme d'utilisation des moyens navals, avec une grande capacité d'adaptation, de négociation avec des 
interlocuteurs multiples et une grande capacité d'initiative. Il poussa d'ailleurs celle-ci jusqu'à susciter la constitution d'armements, comme en 1697 pour celui dirigé par Duguay-Trouin contre le convoi de Bilbao, et à y prendre des participations, quitte à franchir ainsi les limites du « conflit d'intérêts » - ce que lui reprocha Pontchartrain en y entraînant nombre de ses collaborateurs, tel Bordenave, " enseigne de port » et homme d'affaires entreprenant. Mais cette formule hybride des armements mixtes tendait par nature à brouiller les lignes entre public et privé, car, s'agissant cette fois du partenaire "privé », on s'aperçoit que la plupart des signataires des traités passés avec le roi étaient des officiers de la Marine royale, à l'origine du projet, tels Nesmond, Pointis, Des Augiers, Dandennes, et à partir de 1702 Duguay-Trouin, intégré dans le corps des officiers de la Royale depuis 1697. "Intéressés » aux armements dont ils assuraient la codirection avant d'en prendre le commandement, ces marinspromoteurs étaient en fait la figure de proue de groupes d'intérêts privés, structurés en société, qui avaient négocié le traité de prêt et fournissaient les capitaux permettant le montage de l'opération. L'on reste un peu sur sa faim cependant quant à l'analyse de ce volet "privé » des armements mixtes, qu'il s'agisse des structures et mécanismes de drainage des capitaux investis (pour lesquels Jean Meyer avait émis l'hypothèse d'un banc d'essai de la société par actions), ou du profil social des financeurs de ces grandes opérations spéculatives à haut risque. On y trouve certes des investisseurs issus des villes portuaires, comme le groupe négociant malouin animé par Luc Trouin de la Barbinais qui soutint les entreprises de Duguay-Trouin pendant vingt ans, ou comme Du Val d'Eprémesnil au Havre ; et J.-Y. Nerzic met en évidence le rôle important joué par le marchand de bois et fournisseur de la Marine François Saupin, installé à Brest et promoteur des armements de Dandennes en 1695-1697, qui, à cet égard, furent bien des armements "brestois", par la localisation du centre de décision. Mais on devine seulement le rôle majeur qu'ont joué dans le financement de ces entreprises hors norme les milieux de Versailles et de Paris, des «Grands» de la Cour aux ministres (Seignelay), hauts fonctionnaires, gens de finances et banquiers (Samuel Bernard, Jean Nicolas...), sans qu'une analyse systématique en ait été menée. Cela eût exigé, il est vrai, le recours à d'autres sources, notariales notamment, fort lourdes et justifierait une autre thèse sur le sujet, qui n'est paradoxal qu'en apparence, de «Paris (et Versailles) pôle d'armement corsaire » sous Louis XIV!

5 Un effort d'armement considérable donc, mais pour quels résultats? À partir du dépouillement des jugements du Conseil des Prises, J.-Y. Nerzic a également effectué un recensement quasi exhaustif des prises (et rançons) effectuées durant chacune des deux guerres, qui constitue un précieux élément d'appréciation de l'efficacité de cette forme de course. Observons cependant que la seule mesure physique du nombre des prises, dont la valeur pouvait varier de 1 à 100 selon qu'il s'agisse d'une barque chargée de harengs ou d'un indiaman n'a qu'un intérêt relatif, seule comptant vraiment en la matière une estimation en valeur des prises, qu'aurait peut-être permis le recours à la comptabilité du dixième prélevé par l'amiral, déjà utilisée par John S. Bromley. Seule exception, mais précieuse, grâce au fonds Ouesset qui conserve l'ensemble de la comptabilité de l'armement Pointis en 1697, il a pu établir une évaluation précise des résultats financiers de l'expédition hors norme contre Carthagène qui, pour un investissement de 1100000 livres, dégagea plus de 10 millions de livres de produit brut, et $314 \%$ de profit net pour les intéressés. Beau feu d'artifice en conclusion de la guerre de la Ligue d'Augsbourg, comme le fut l'expédition de Rio à la fin de celle de Succession, qui dégagea $92 \%$ de profit. Mais ces deux réussites spectaculaires (et il y en eut 
quelques autres, que pointe l'auteur, comme lors de la capture d'indiamen par DuguayTrouin et Nesmond en 1695) ne suffisent pas à démontrer que cette forme d'armement en course ait dégagé un bilan d'ensemble positif pour les investisseurs privés, faute d'une estimation globale comparée, en valeur, de l'investissement engagé et du produit total des prises effectuées. De même demeure-t-il difficile de porter un jugement définitif sur l'efficacité politico-militaire de cette modalité particulière de la stratégie navale de "guerre au commerce » adoptée par le pouvoir royal en 1694-1695, même si ce travail apporte un peu d'eau au moulin des thèses de Vauban.

6 Le chantier demeure donc ouvert sur ce pan d'histoire maritime exceptionnel, mais il faut reconnaître à ce travail considérable, à tous égards « utile » et sur plusieurs points novateur, d'en avoir solidement bâti les fondations. 